\title{
Multivariate Analysis of the Globular Clusters in M87
}

\author{
Sukanta Das $^{1}$, Tanuka Chattopadhayay ${ }^{1,3}$ and Emmanuel Davoust ${ }^{2,4}$ \\ ${ }^{1}$ Department of Applied Mathematics, University of Calcutta, 92 A.P.C Road, Calcutta 700009, India \\ ${ }^{2}$ Institut de Recherche en Astrophysique et Planétologie, Université de Toulouse/CNRS, 14 Avenue Edouard Belin, 31400 Toulouse, France \\ ${ }^{3}$ Email: tanuka@iucaa.ernet.in \\ ${ }^{4}$ Email: edavoust@irap.omp.eu
}

(Received June 13, 2015; AccePted September 30, 2015)

\begin{abstract}
An objective classification of 147 globular clusters (GCs) in the inner region of the giant elliptical galaxy M87 is carried out with the help of two methods of multivariate analysis. First, independent component analysis (ICA) is used to determine a set of independent variables that are linear combinations of various observed parameters (mostly Lick indices) of the GCs. Next, $K$-means cluster analysis (CA) is applied on the independent components (ICs), to find the optimum number of homogeneous groups having an underlying structure. The properties of the four groups of GCs thus uncovered are used to explain the formation mechanism of the host galaxy. It is suggested that M87 formed in two successive phases. First a monolithic collapse, which gave rise to an inner group of metal-rich clusters with little systematic rotation and an outer group of metal-poor clusters in eccentric orbits. In a second phase, the galaxy accreted low-mass satellites in a dissipationless fashion, from the gas of which the two other groups of GCs formed. Evidence is given for a blue stellar population in the more metal rich clusters, which we interpret by Helium enrichment. Finally, it is found that the clusters of M87 differ in some of their chemical properties ( $\mathrm{NaD}$, TiO1, light-element abundances) from GCs in our Galaxy and M31.
\end{abstract}

Keywords: galaxies: formation - galaxies: star clusters: general - methods: statistical

\section{INTRODUCTION}

The details of galaxy formation and evolution remain one of the great unsolved problem in modern astrophysics. Giant elliptical galaxies at the centre of galaxy clusters are of strong interest as they provide case studies for galaxy formation theory. On the other hand, GCs are excellent tracers of galaxy halos. Indeed, some $50 \%$ of the stars in our Galaxy may have originated in GCs (Martell \& Grebel 2010). GCs are known to form in vigorous star formation events (Brodie \& Strader 2006 and references therein) and were an important mode of star formation in the early universe (Muratov \& Gredin 2010). It is possible that all stars may have originally formed in star clusters which are only later dispersed into the smooth stellar halos we see today (Lada \& Lada 2003). For making progress in galaxy formation theory, a holistic approach is required, which involves compositional as well as kinematical properties of GCs in giant elliptical galaxies. A landmark study (Emsellem et al. 2007) suggested that a fundamental dichotomy exists between dynamically 'slow' and 'fast' rotating elliptical galaxies. These observations prompted new interpretations and theoretical models to explain this difference (Krajnovic et al. 2008; Jesseit et al. 2009). Proctor et al. (2009) suggested that the rotator class may change when the kinematics are probed beyond the inner regions.

According to various studies, classical formation of galaxies have been proposed along five major lines: (i) the monolithic collapse model, (ii) the major merger model, (iii) the multiphase dissipational collapse model, (iv) the dissipationless merger model and (v) accretion and in situ hierarchical merging. But no model is globally acceptable over others.

In this context, one is tempted to make a detailed study of an archetype of each category of elliptical galaxy. In previous papers (Chattopadhyay et al. 2009; Chattopadhyay, Mondal, \& Chattopadhyay 2013), we studied the GCs of NGC5128, which is a slowly rotating elliptical galaxy. In the present paper we study the GCs of M87, which is a strongly rotating elliptical galaxy, located at the centre of the Virgo cluster. It has a large population of GCs with well-known kinematic (Huchra \& Brodie 1987; Cohen \& Ryzhov 1997; Hanes et al. 2001; Côté et al. 2001; Strader et al. 2011), photometric (Strom et al. 1981; Strader et al. 2011) and chemical properties (Mould, Oke, \& Nemec 1987; Mould et al. 1990; Cohen, Blakeslee, \& Ryzhov 1998). To this end, we carry out a multivariate analysis of the Lick indices, metallicities and radial distances of the GCs. First, we use independent component 

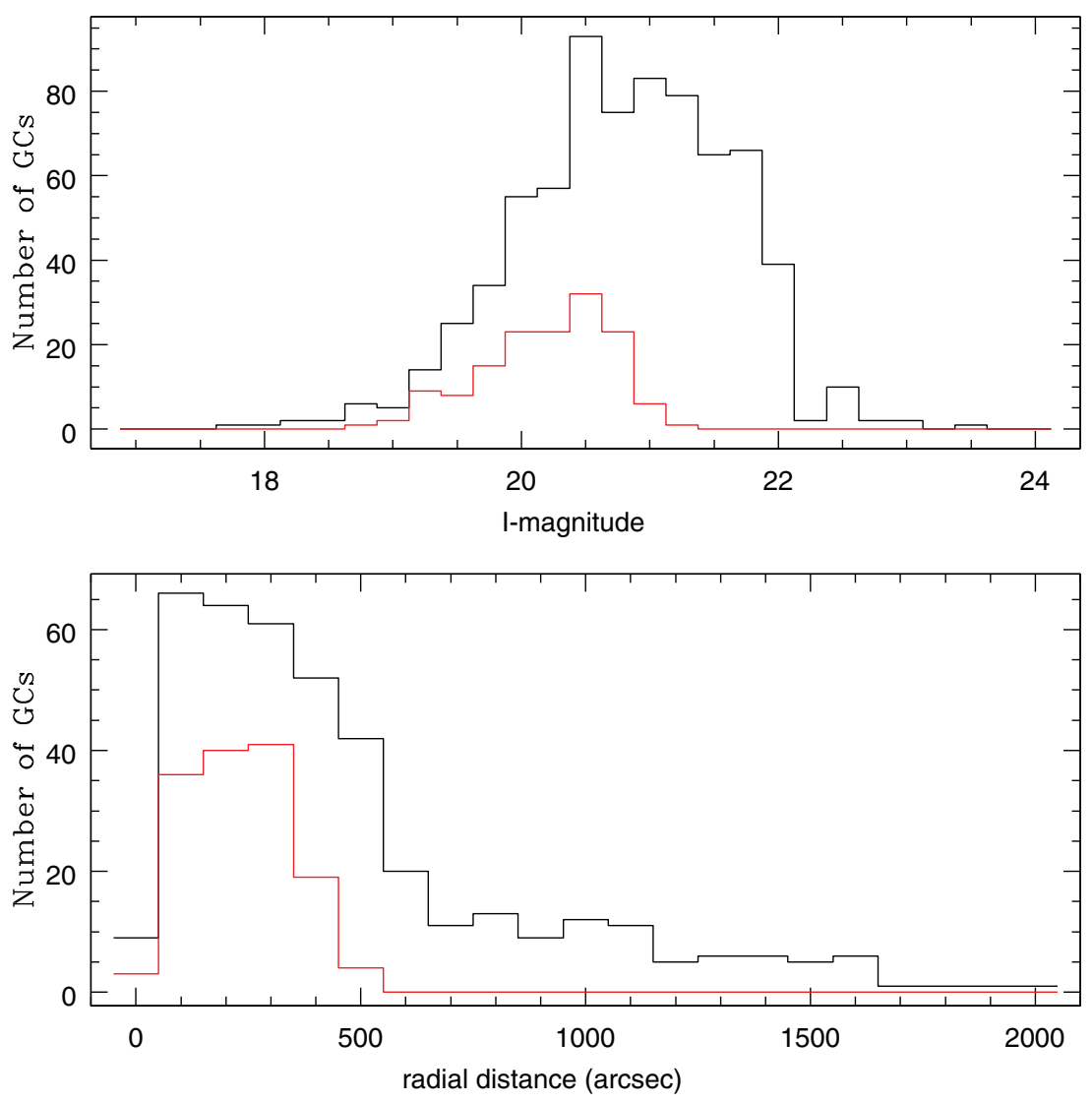

Figure 1. Comparison of the histograms of $i_{\text {mag }}$ and R of the GCs of our sample (red solid line) and the sample of Strader et al. (2011) (black solid line).

analysis (hereafter ICA), suitable for non-Gaussian data sets to search for the independent components (hereafter IC). The number of such components, which are linear combinations of parameters, is equal to the number of parameters ( $p$, say). Since only a few (say, $m \ll p$ ) of the IC components may explain a larger percentage of variation in the data, one can take only those $\mathrm{m}$ components instead of all $\mathrm{p}$ components. Then the GCs are classified on the basis of those $m$ ICs using another exploratory data analytic method, namely $K$-means CA (Chattopadhyay et al. 2009; Chattopadhyay, Sharina, \& Karmakar 2010; Chattopadhyay et al. 2012; Chattopadhyay \& Karmakar 2013; Chattopadhyay, Mondal, \& Chattopadhyay 2013) to find the homogeneous groups. In the end, the properties of the homogeneous groups of GCs allow us to propose a possible scenario for the formation of the GCs and their host galaxy.

The data sets used are presented in Section 2. Section 3 describes different methods used in the study. The results and interpretations have been included in Section 4.

\section{DATA SET}

The Lick indices $(H \beta, \mathrm{Mg} 1, \mathrm{Mg} 2, \mathrm{Mgb}, \mathrm{Fe} 5270, \mathrm{Fe} 5335$, $\mathrm{NaD}$ and $\mathrm{TiO} 1)$ and metallicities $(\mathrm{Fe} / \mathrm{H})$ used in the present analysis were taken from Cohen et al. (1998). From the orig- inal sample of 150 GCs we removed the GCs with identification numbers 5024 and 5026, because they are redundant with 978 (Hanes et al. 2001), as well as 321 which is a star (Strader et al. 2011), thus reducing the sample to 147 GCs. In the latter paper, the identification number given by Cohen et al. (1998) is preceded by ' $\mathrm{S}$ '. The velocities $v_{\text {rad }}$, half-light radii $r_{h}, i$ magnitudes $\left(i_{\mathrm{mag}}\right)$ and $(g-r)$ and $(g-i)$ colours were taken from Strader et al. (2011). The radial distances $R$ and position angles $\psi$ were derived from the coordinates listed in Strader et al. (2011), assuming that the centre of the system of GCs is also that of the galaxy, which is at $\alpha_{c}=12$ h $30 \mathrm{~m} 49.42 \mathrm{~s}$ and $\delta_{c}=12 \mathrm{~d} 23^{\prime} 28.044^{\prime \prime}$ (Lambert \& Gontier 2009).

It is to be noted that our sample size is small compared to the recently observed samples by various authors (e.g. Strader et al. 2011). We have thus plotted the histograms of $i$ magnitudes and radial distances of our sample and have compared with the sample used by Strader et al. (2011) (Figure 1). Now Strader et al. (2011) have found that the bright and faint GCs have the same velocity dispersions (sigma $=340$ and $339 \mathrm{kms}^{-1}$ ). In their Section 6.3.4, they find that there is no strong trend with magnitude for the red GCs. For the blue GCs, there is perhaps an avoidance of the systematic velocity, although marginal. The conclusion is that the bright and faint compact blue GCs belong to the same population. So we can 
safely say that, if the kinematics are an indicator of the origin of GCs, then our sample should be enough to determine the origin of the inner GCs.

For comparison purposes, we used the Lick indices of 313 old clusters in M31 (Schiavon et al. 2012), those of 47 GCs in 4472 (Cohen, Blakeslee, \& Côté 2003) and 33 GCs in NGC 4636 (Park et al. 2012).

\section{STATISTICAL METHODS}

\subsection{Shapiro-Wilk test}

The choice of statistical methods to apply to a set of data depends on its Gaussian or non-Gaussian nature. We have thus tested the Gaussianity of our data set by the ShapiroWilk test (1965). According to this test, the null hypothesis is that the data set is Gaussian. The test statistic W is defined as $\mathrm{W}=\sum_{i=1}^{n} a_{i} x_{i}^{2} / \Sigma_{i=1}^{n}\left(x_{i}-\bar{x}\right)^{2}$ where $n$ is the number of observations, $x_{i}$ are the ordered sample values and $a_{i}$ are constants generated from means, variances, and covariances of the order statistics of a sample of size $n$ from a normal distribution. In our case the data set is multivariate, hence we have used the multivariate extension of the Shapiro-Wilk test. We found that the $p$ value of the test is $1.327 \times 10^{-11}$, which is very small. Thus, the null hypothesis is rejected at a 5\% level of significance. We conclude that the present data set is non-Gaussian in nature.

\subsection{Independent component analysis}

While Principal Component Analysis (PCA) applies to Gaussian data sets, ICA applies to non-Gaussian data sets. ICA is a dimension reduction technique, i.e. it reduces the number of observable parameters $p$ to a number $m(m \ll p)$ of new parameters, where these new parameters are the linear combinations of $p$ parameters, such that these $m$ parameters are mutually independent. Mathematically speaking, let $X_{1}, X_{2}, \ldots, X_{p}$ be $p$ random vectors (here $p$ parameters) and $n$ (here 147) be the number of observations of each $X_{i}(i=$ $1,2, \ldots, p)$.

Let

$$
X=A S,
$$

where $S=\left[S_{1}, S_{2}, \ldots, S_{p}\right]^{\prime}$ is a random vector of hidden components $S_{i}(i=1,2, \ldots, n)$ such that $S_{i}$ are mutually independent and $\mathrm{A}$ is a non-singular matrix. The goal of ICA is to estimate $A$ and to find $S$ by inverting $A$, i.e.

$$
S=A^{-1} X \quad \text { i.e. } S=W X \text {. }
$$

Under ICA, we find the components $\left[S_{1}, S_{2}, \ldots, S_{n}\right]$ those are independent by finding an unmixing matrix $\mathrm{W}$ in such a way that the covariance between any two nonlinear functions $g_{1}\left(S_{i}\right)$ and $g_{2}\left(S_{j}\right)$ for $i \neq j$ is zero i.e. the ICs are nonlinearly uncorrelated (for more details, see Comon (1994); Chattopadhyay Mondal, \& Chattopadhyay, (2013) and references therein).
At present, there is no better method available to automatically determine the optimum number of ICs. In this paper, the number of ICs is determined by the number of Principal Components (PCs) chosen (Albazzaz and Wang, 2004). To reduce the number of components $S_{i}$ from $p$ to $m(m \ll p)$, one is to perform PCA (Babu et al. 2009; Chattopadhyay \& Chattopadhyay 2007; Fraix-Burnet et al. 2010; Chattopadhyay et al. 2010). In this method also $Y_{i}$ 's $(i=1,2, \ldots, p)$ vectors are found, which are linear combinations of $X_{i}^{\prime}$ 's $(i=1,2, \ldots, p)$ such that $Y_{i}^{\prime}$ 's are uncorrelated. The number of (initially $p$ ) components is reduced to $m$ by taking those $Y_{i}^{\prime}$ 's $(i=1,2 \ldots, m)$ for which the corresponding eigenvalues $\lambda_{i} \sim 1$.

In the present work, we have first performed PCA to find the significant number of IC components to be chosen. PCA applies to Gaussian data, but the present data set is nonGaussian, so we performed ICA. In PCA, the maximum variation with significantly high eigenvalue (viz. $\lambda \sim 1$ ) was found to be almost $78 \%$ for three PCA components. Hence, we have chosen three IC components for the $\mathrm{CA}$. The parameters chosen for ICA are the Lick indices $H \beta, \mathrm{Mg} 1, \mathrm{Mg} 2$, $\mathrm{Mgb}, \mathrm{Fe} 5270, \mathrm{Fe} 5335, \mathrm{NaD}$, TiO, the metallicity $\mathrm{Fe} / \mathrm{H}$, the photometric parameters magnitude $i_{\text {mag }}$ and colours $(g-r)$, $(g-i)$ and the projected galactocentric distance $R$ (in $\operatorname{arcsec}$ ).

\section{3. $K$-means cluster analysis}

$K$-means CA is a multivariate technique for finding homogeneous groups in a data set giving information of the underlying structure in this data set. In this method one finds $k$ groups, provided each group contains an object and each object belongs to exactly one group. Hence, the maximum and minimum number of possible groups are $k=n$ and $k=1$, respectively. The algorithm is as follows :

(i) All the objects are divided into $k$ (given) groups in a random manner.

(ii) Any group is selected and any particular object of that group is taken first. Then the parametric distance (here the parameters are three IC components) from the chosen object is computed for the remaining objects. If the distance between objects in the group is greater than that for objects in other groups, they are interchanged.

(iii) Process (ii) is applied for all objects and for all groups.

(iv) Steps (i) and (iii) are continued until there is no further change.

The $k$ groups thus found are coherent in nature.

The optimum value of $k$ is found as follows. First one finds groups for $k=1,2,3, \ldots$ etc. Then a distance measure $\mathrm{d}_{k}$ is computed by

$$
\mathrm{d}_{k}=(1 / p) \min _{x} E\left[\left(x_{k}-c_{k}\right)^{\prime}\left(x_{k}-c_{k}\right)\right],
$$

which is the distance of $x_{k}$ vector (values of parameters) from the centroid $c_{k}$ of the corresponding group. The optimum value of $k$ is that for which $J_{k}=\left(\mathrm{d}_{k}^{-p / 2}-\mathrm{d}_{k-1}^{\prime-p / 2}\right)$, 
Table 1. Discriminant analysis for $K$-means classification: G1, G2, G3, and G4 are groups found by $K$-means cluster analysis and $G 1^{*}$, $G 2^{*}, G 3^{*}$, and $G 4^{*}$ are groups found by discriminant analysis.

\begin{tabular}{lrrrr}
\hline \hline DA groups & $\mathrm{G} 1$ & $\mathrm{G} 2$ & $\mathrm{G} 3$ & $\mathrm{G} 4$ \\
\hline$G 1^{*}$ & 51 & 2 & 3 & 0 \\
$G 2^{*}$ & 2 & 52 & 1 & 1 \\
$G 3^{*}$ & 2 & 0 & 23 & 0 \\
$G 4^{*}$ & 0 & 0 & 0 & 10 \\
Total & 55 & 54 & 27 & 11 \\
\hline \hline
\end{tabular}

is maximum (Sugar \& James 2003). In the present case, $k=4$.

For CA, the number of parameters are three IC components chosen as previously described. The optimum number of groups is selected objectively by $k$-means clustering (MacQueen 1967), together with the method developed by Sugar and James (2003). The optimum number is $k=4$. The groups are labelled G1, G2, G3, and G4.

For testing the robustness of the classification, we have proceeded in the following manner. In the original scheme, the number of parameters is 13 . First, we have constructed the variance covariance matrix of the parameters and selected the parameters having maximum variances e.g. here $H_{\beta}, \mathrm{Mgb}$, $\mathrm{Fe} 5270, \mathrm{Fe} 5335, \mathrm{NaD}, \mathrm{Fe} / \mathrm{H}, i_{\text {mag }}$, R have variances greater than 0.25 and the remaining ones, $\mathrm{Mg} 1, \mathrm{Mg} 2, \mathrm{TiO} 1, g-r, g-i$ have variances $\sim 0.0$. Therefore, we have done the analysis with the above eight parameters and have obtained exactly the same groups with no variation at all. So, we can at once say that our classification is robust with respect to the parameters which are responsible for the maximum variation.

Regarding the uncertainties on the various parameters, the method we have used is totally exploratory (i.e. no underlying distribution is assumed). Hence, it is not possible to see the effect of error in such type of analysis directly. What we can do at most is to change the values of the parameters within the range permitted by the error bars and redo the analysis. We have tested it for that for few values of the parameters and found the same groups. Alternatively, once the optimum classification (clustering) is obtained, one can use a process called discriminant analysis (Johnson \& Wichern 1998) to verify the acceptability of the classification of different GCs. In this standard procedure, using the probability density functions in parameter space for the different clusters, one can assign an object (in this case, a GC) to be a member of a certain class. If the original classification is robust, then every GC should be classified again as a member of the same class that it was before. If a significant number of objects are not reclassified then that means that the original classification is not stable and hence not trustworthy. Table 1 shows the result of a discriminant analysis, where the columns represent how the GCs of a cluster were assigned by the analysis. The fraction of correct classifications is 0.925 , which im- plies that the classification is indeed robust by computing classification/misclassification probabilities for the GCs.

\subsection{Levenberg-Marquardt Algorithm}

This algorithm is used to find the most probable mean rotation curve for each group, assuming that they have a solid-body rotation around the centre of the galaxy. This is a reasonable assumption for the two innermost groups, G1 and G4, because the galaxy itself is in solid-body rotation at least to a radius of 225 arcsec (Cohen \& Ryzhov, 1997). For the two other groups, G2 and G3, we also tried a rotation curve that flattens beyond 225 arcsec, but the data were better adjusted by a solid-body rotation curve (also see Figure 7 of Kissler-Patig \& Gebhardt 1998).

The rotation amplitudes $v_{\text {rot }}(=\Omega R)$ and the position angles $\left(\psi_{0}\right)$ of the axes of rotation (East to North) of the different groups $\mathrm{G} 1-\mathrm{G} 4$, (found in the CA) are computed by the relation

$$
v_{\text {rad }}(\psi)=v_{\text {sys }}+\Omega R \sin \left(\psi-\psi_{0}\right),
$$

(Côté et al. 2001; Richtler et al. 2004; Woodley et al. 2007), where $v_{r}$ is the observed radial velocity, $v_{\text {sys }}$ is taken as the recession velocity of the galaxy, which is $1287 \mathrm{~km} \mathrm{~s}^{-1} .^{1} R$ is the projected distance of each GC and $\psi$ is its position angle, measured East to North. We have used Levenberg-Marquardt fitting method (Levenberg 1944; Marquardt 1963) to solve for $v_{\text {rot }}$ and $\psi_{0}$. They are listed in Table 2 for G1-G4. Now kinematic data sets for G1-G4 are small and for such situation Monte Carlo simulations are used to increase the data sets to have a more convincing result. Monte Carlo simulation needs distributional assumption and here no well known bivariate distribution is fitting with the data well. So, we have taken several bootstrap samples and have computed the mean values with standard errors of the rotation parameters. The errors are small as seen from Table 2 .

\section{RESULTS AND INTERPRETATION}

\subsection{Properties of globular clusters in the four groups}

The CA divided the GCs of M87 into four groups. We did not use the kinematical data $\left(v_{\text {rad }}\right.$ and $\left.\psi\right)$ in the statistical analysis. Nor did we use $\mathrm{Mgb} / \mathrm{Fe}$. The latter is an indicator of the abundance in light elements. But we did use these parameters for interpreting the results.

The results of the analysis are summarised in Table 2, which lists the mean values of all the parameters with standard errors for the four groups. The mean velocities of rotation and mean position angles have been computed by the Levenberg-Marquardt algorithm.

To show how the groups are separated, we have plotted three ICs (viz. IC1, IC2 and IC3) for the groups G1-G4 
Table 2. Mean values of various parameters of G1-G4 together with kinematics.

\begin{tabular}{lcccc}
\hline \hline Parameter & $\mathrm{G} 1$ & $\mathrm{G} 2$ & $\mathrm{G} 3$ & $\mathrm{G} 4$ \\
\hline No. of GCs & 55 & 54 & 27 & 11 \\
$H_{\beta}$ & $2.0271 \pm 0.0735$ & $1.9489 \pm 0.08$ & $2.0607 \pm 0.08$ & $1.238 \pm 0.13$ \\
$\mathrm{Mg} 1$ & $-0.00907 \pm 0.004$ & $-0.01204 \pm 0.004$ & $-0.0323 \pm 0.005$ & $0.1015 \pm 0.01$ \\
$\mathrm{Mg} 2$ & $0.08618 \pm 0.007$ & $0.09270 \pm 0.007$ & $0.01981 \pm 0.009$ & $0.2781 \pm 0.02$ \\
$\mathrm{Mgb}$ & $2.123 \pm 0.115$ & $2.537 \pm 0.12$ & $1.1511 \pm 0.09$ & $4.765 \pm 0.19$ \\
$\mathrm{Fe} 5270$ & $2.019 \pm 0.102$ & $1.9381 \pm 0.073$ & $0.711 \pm 0.13$ & $2.876 \pm 0.17$ \\
$\mathrm{Fe} 5335$ & $1.5482 \pm 0.09$ & $1.6481 \pm 0.08$ & $0.5448 \pm 0.09$ & $2.405 \pm 0.24$ \\
$\mathrm{NaD}$ & $1.5122 \pm 0.09$ & $1.6646 \pm 0.001$ & $1.03 \pm 0.11$ & $5.125 \pm 0.52$ \\
$\mathrm{TiO} 1$ & $0.01169 \pm 0.002$ & $0.0172 \pm 0.04$ & $0.00081 \pm 0.002$ & $0.03682 \pm 0.003$ \\
$\mathrm{Fe} / \mathrm{H}$ & $-0.9807 \pm 0.05$ & $-0.832 \pm 0.07$ & $-1.6289 \pm 0.08$ & $-0.06 \pm 0.06$ \\
$\mathrm{Mgb} / \mathrm{Fe}$ & $1.2318 \pm 0.0628$ & $1.4184 \pm 0.0513$ & $3.49 \pm 1.36$ & $1.8543 \pm 0.0925$ \\
$i_{\text {mag }}$ & $20.229 \pm 0.06$ & $20.077 \pm 0.006$ & $20.476 \pm 0.06$ & $20.002 \pm 0.13$ \\
$g-r$ & $0.60291 \pm 0.005$ & $0.61833 \pm 0.01$ & $0.55037 \pm 0.006$ & $0.72 \pm 0.02$ \\
$g-i$ & $0.8727 \pm 0.01$ & $0.9076 \pm 0.01$ & $0.7837 \pm 0.01$ & $1.0855 \pm 0.04$ \\
$R$ & $147.73 \pm 8.36$ & $338.03 \pm 8.92$ & $234.2 \pm 17.9$ & $146.4 \pm 19.6$ \\
$v_{\text {rot }}$ & $30.92 \pm 7.42$ & $232.96 \pm 69.73$ & $40.93 \pm 10.34$ & $249.02 \pm 19.11$ \\
$\psi_{o}$ & $92.58 \pm 14.27$ & $265.38 \pm 19.41$ & $167.20 \pm 13.59$ & $217.14 \pm 50.65$ \\
\hline \hline
\end{tabular}

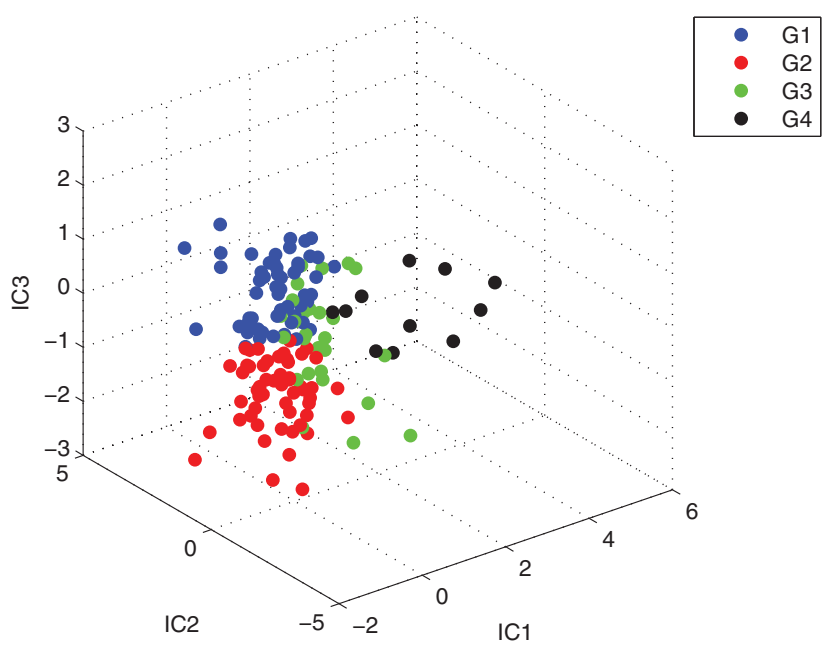

Figure 2. Plot of IC1, IC2, and IC3 of G1(blue circles), G2(red circles), G3(green circles), and G4 (black circles) groups of GCs.

found in our analysis (Figure 2). That figure shows that the groups are well separated in IC space.

We first investigate how the different groups differ in their Lick index values, and how they compare in this respect with the simple stellar population models of Thomas, Maraston, \& Johansson (2011). As shown in Figures 3-5, G3 has the lowest values of Fe5270, NaD,TiO, and Mgb, while G4 has the highest values of these Lick indices, and G1 and G2 are in between. These two latter groups differ in that G1 is marginally less chemically evolved than G2 (these two groups also differ in their spatial distribution and in their kinematics). In other words, the order of increasing overall chemical evolution is G3, G1, G2, and G4. The Lick index $\mathrm{H} \beta$ is an age indicator, higher values meaning younger ages,

${ }^{1}$ Côté et al. (2001) adopted $v_{\text {sys }}=1350 \mathrm{~km} \mathrm{~s}^{-1}$.

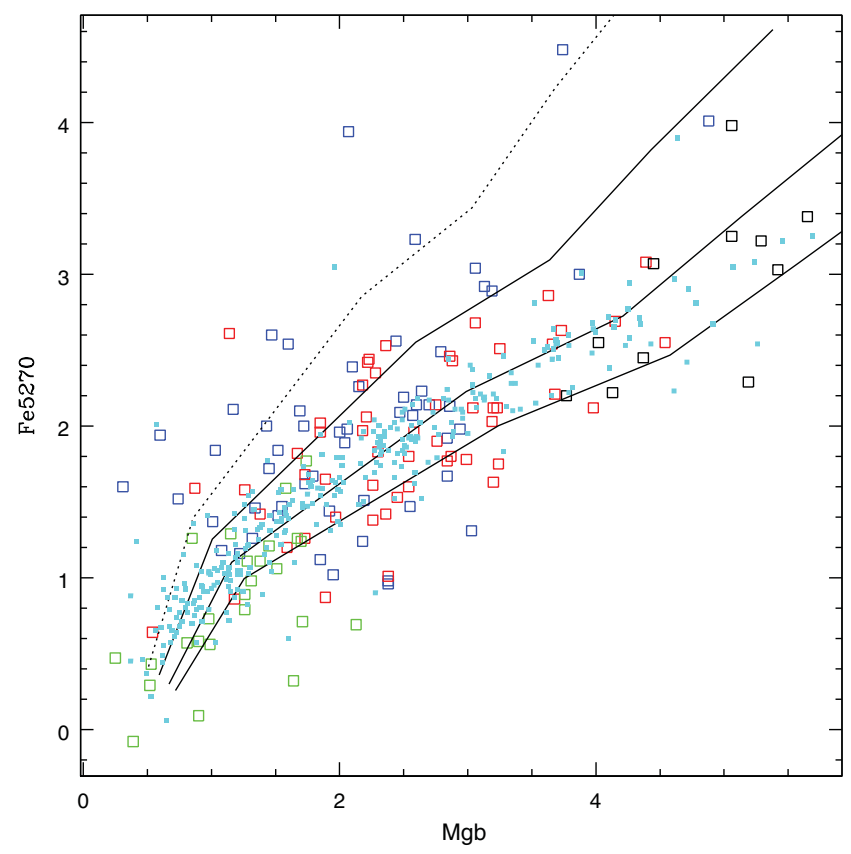

Figure 3. Fe 5270 versus Mgb for G1-G4. Blue open squares for G1, red open squares for $\mathrm{G} 2$, green open squares for $\mathrm{G} 3$, black open squares for G4, cyan filled squares for GCs of M31. Black lines are stellar population models of Thomas et al.(2011) for an age of $12 \mathrm{Gyr}$, a dotted line for $\alpha / F e$ $=-0.3$ and solid lines are for $\alpha / \mathrm{Fe}=0.0,0.3$, and 0.5 respectively from left to right.

but this index is also sensitive to the colour of the Horizontal Branch (HB), higher values meaning a bluer HB. A bluer HB in turn can be due to He-enriched stars. So the interpretation of Figure 6, which shows the run of $\mathrm{H} \beta$ versus Mgb, is best done with the help of stellar population models, namely those of Thomas et al. (2011). The data are well fit by models of 12 Gyr, albeit with a large scatter suggesting a variety of $\mathrm{HBs}$ and/or of $\alpha / \mathrm{Fe}$. An alternative explanation in terms of stellar populations with younger ages is not possible because 


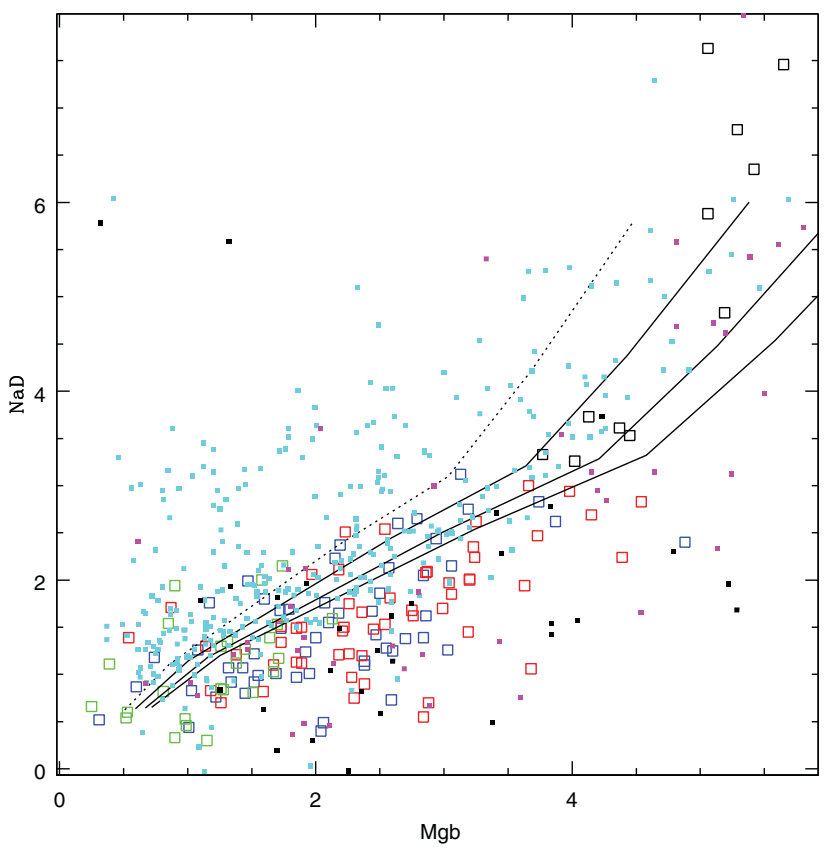

Figure 4. $\mathrm{NaD}$ versus $\mathrm{Mgb}$ for $\mathrm{G} 1-\mathrm{G} 4$ and for $\mathrm{GCs}$ of $\mathrm{M} 31$ (small cyan squares), NGC4472 (small magenta squares), NGC4636 (small black squares). The colours and symbols are the same as in Figure 3. $\mathrm{NaD}$ is higher in the GCs of the spiral galaxy than in those of the elliptical galaxies.

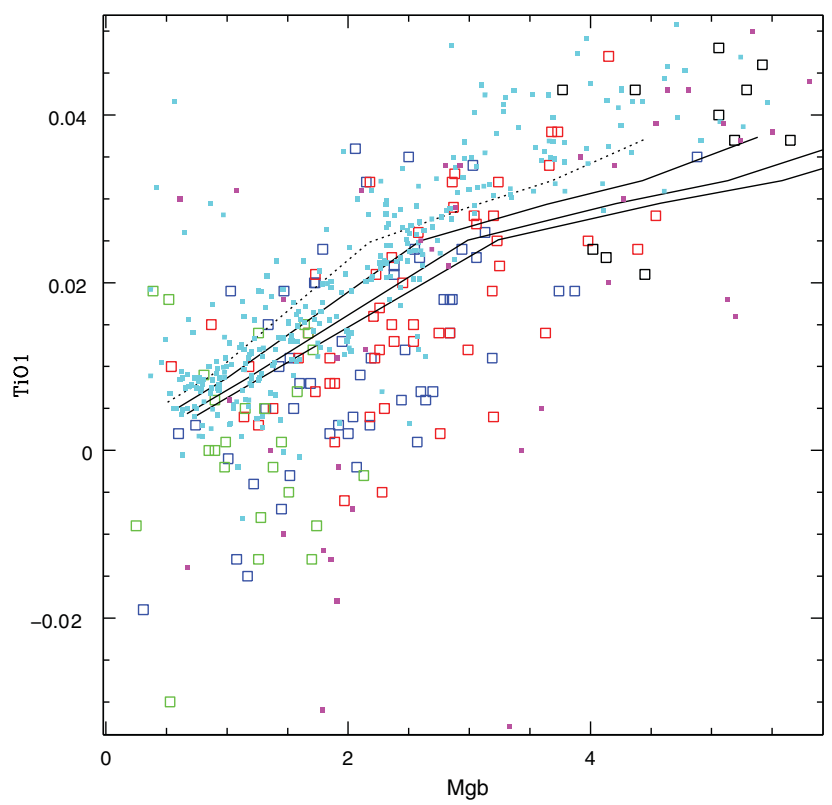

Figure 5. TiO1 versus Mgb for G1-G4 along with GCs of M31 and NGC4472. The colours and symbols are the same as in Figure 4. TiO1 is higher in the GCs of the spiral galaxy than in those of the elliptical galaxies.

it would not be compatible with the observed broad-band colours (see below).

We next investigate the colours of the stellar populations in the GCs of M87. The colour-colour diagram for the different groups is shown in Figure 7. There is a progressive

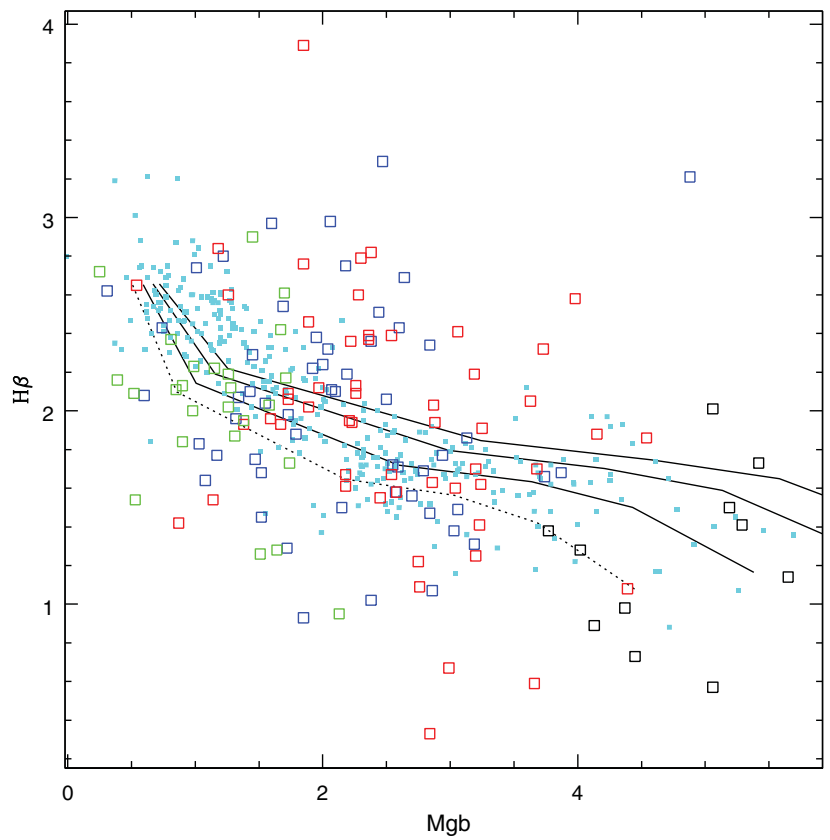

Figure 6. $H \beta$ vesus Mgb for G1-G4. The colours and symbols are same as in Figure 4. The large spread in $H \beta$ in G1-G4 can be due to a spread in $\alpha / \mathrm{Fe}$ and/or in colour of the Horizontal Branch.

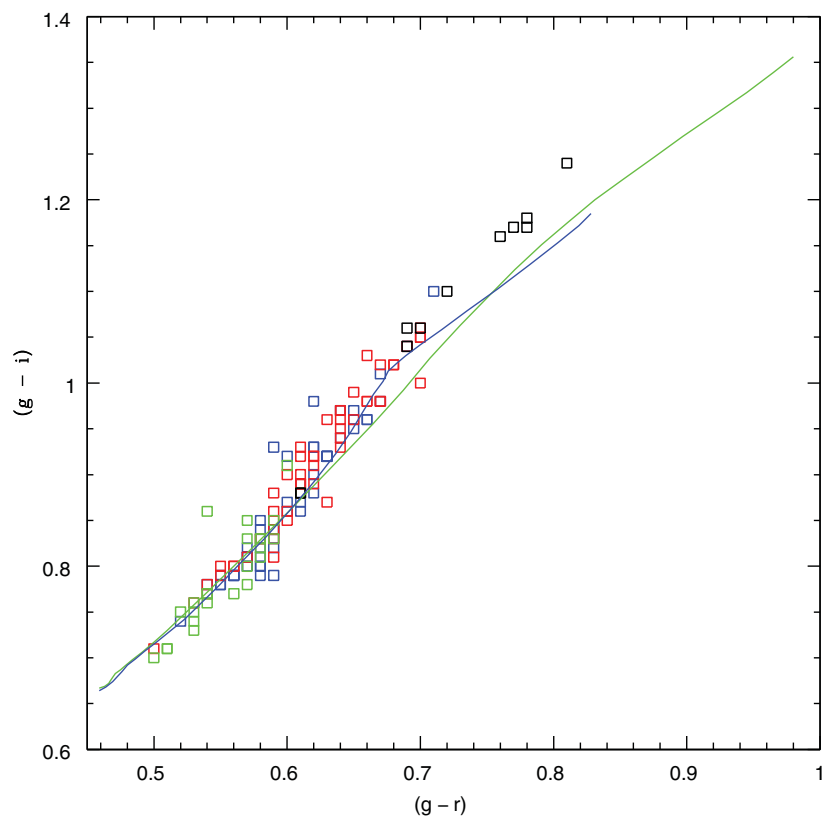

Figure 7. $(g-i)$ versus $(g-r)$ for G1-G4. The colours and symbols are the same as in Figure 3. The green and blue solid lines are Yonsei models for an age of $12 \mathrm{Gyr}$, and for 0 and $70 \%$ secondary stellar populations respectively.

reddening of the populations, from G3 (green) to G1 (blue) followed by G2 (red) and G4 (black). This is predicted by models of synthetic stellar populations as a result of increasing age and/or metallicity. Since the models of Thomas et al. (2011) do not predict the evolution of broad-band colours, we adjusted Yonsei models of simple stellar populations (Chung et al. 2013) to the data (Figure 8). The models for 


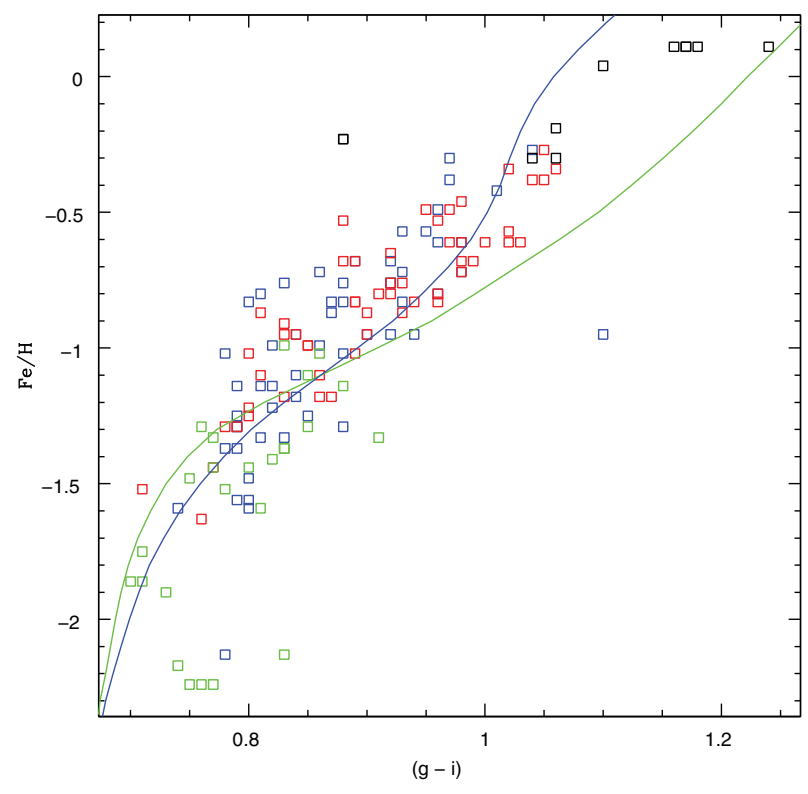

Figure 8. $[\mathrm{Fe} / \mathrm{H}]$ versus $(g-i)$ for $\mathrm{G} 1-\mathrm{G} 4$. The colours and symbols are same as that for Figure 3. The green and blue lines are Yonsei stellar population models (Chung et al. 2013) for an age of $12 \mathrm{Gyr}$ and 0 and $70 \% \mathrm{He}$ enhanced secondary populations respectively.

metallicities ranging from $[\mathrm{Fe} / \mathrm{H}]=-2.6$ to 0.6 , an age of 12 Gyr and $\alpha / F e=0.3$ are shown as solid lines, green and blue for 0 and $70 \%$ secondary populations respectively. Changing $\alpha / F e$ will only shift the model down diagonally on the figure, and changing the initial mass function has little effect on the models (see Chung et al. 2013). The comparison of our data to the Galev models (Kotulla et al. 2009), and to the models of Maratson et al. (2003) gave results (not shown) very close to those of the Yonsei model for $0 \%$ secondary populations. For the Maraston models, we converted the CFH-Megcam magnitudes to SDSS using the paper by Betoule et al. (2013).

The colours of the metal-poor GCs (shown on Figure 7) are well fit by the models. On the other hand, the metalintermediate (G1) and metal-rich (G2) GCs can only be explained by a Helium-enriched secondary stellar population (Chung et al. 2013). No Yonsei model population with a realistic age is able to account for the colours of group G4. We have no explanation for the colours of this group, other than that they are unusual. It is not possible to advocate younger stellar populations instead of He-enriched ones to interpret the colours of the groups, because such populations are bluer in both colours, but more so in $(g-i)$. Further evidence for He enhancement in the metal-intermediate and metal-rich GCs of M87 is the far ultraviolet excess detected in the GCs of M87 by Sohn et al. (2006), an excess which increases with metallicity. Kaviraj et al. (2007) interpret this excess by the possible presence of hot HB stars from super-He-rich stellar populations, even though their predicted ages are unrealistically old.

We next compare the properties of the GCs of M87 with those of other galaxies. The GCs of M87 have values of $\mathrm{Fe} 5270$ and $\mathrm{H} \beta$ at a given $\mathrm{Mgb}$ (see Figures 3 and 6) that are

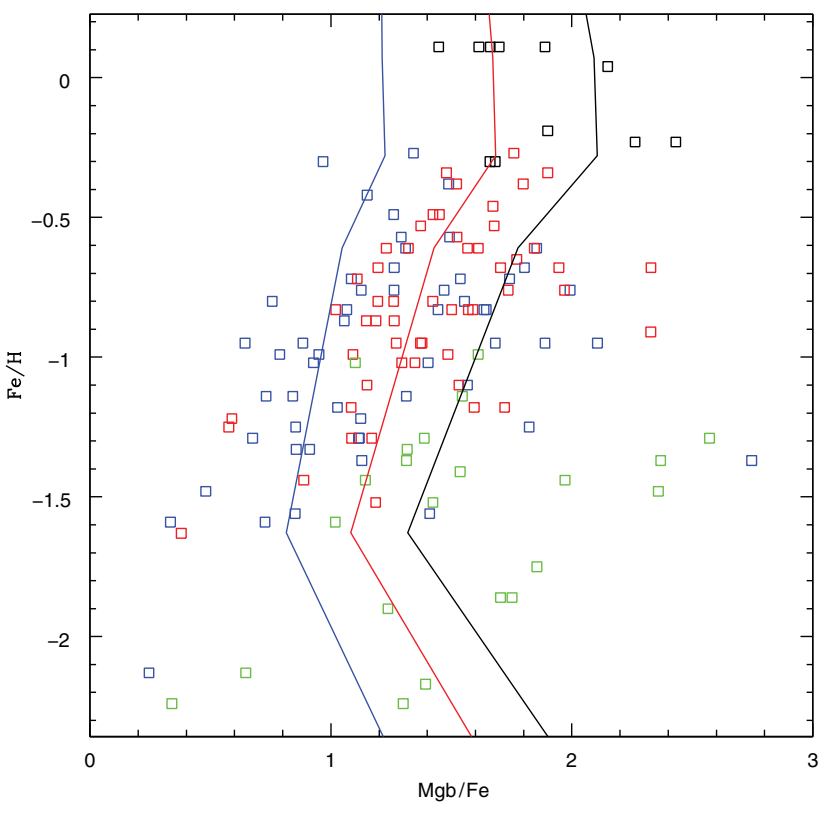

Figure 9. $\mathrm{Fe} / \mathrm{H}$ versus light-element abundance indicator $\mathrm{Mgb} / \mathrm{Fe}$ for $\mathrm{G1}-$ G4. The colours and symbols are same as in Figure 3. The blue, red, and black lines are stellar populations of Thomas et al. (2011) for $\alpha / \mathrm{Fe}=0.0$, 0.3 , and 0.5 respectively. $\alpha / \mathrm{Fe}$ increases with metallicity in $\mathrm{G} 1, \mathrm{G} 2$, and $\mathrm{G} 4$, while in $\mathrm{G} 3$ it has a peculiar behaviour.

comparable to those of M31, but generally lower values of $\mathrm{NaD}$ and $\mathrm{TiO}$ at a given $\mathrm{Mgb}$ (see Figures 4 and 5), except G4. In the latter two figures, the predictions of the simple stellar population models seem to hold the middle ground between the GCs of M87 and of M31. Concerning NaD, the high values observed in M31 might be partly due to interstellar absorption and thus less reliable. We are indebted to the referee for this remark. To better understand the significance of these lower values, we also plotted the same data for GCs of two other elliptical galaxies in Virgo, NGC 4472 and 4636 on Figures 4 and 5. The GCs of these two other ellipticals show the same behaviour as those of M87, suggesting that GCs in elliptical galaxies are different from those of spirals in this respect, except at very high metallicities. We speculate that $\mathrm{Na}$ is underabundant in $\mathrm{G} 1-\mathrm{G} 3$ and that the $\mathrm{Na}-\mathrm{O}$ anticorrelation present in Galactic GCs, interpreted as a result of multiple stellar populations, is only present in G4.

The abundance of light elements tends to decrease with increasing metallicity in the GCs of our Galaxy (Fraix-Burnet, Davoust \& Charbonnel 2009), M31 (Colucci et al. 2012a), and the LMC (Colucci, Bernstein, \& Cohen 2012b), as expected from arguments of nucleosynthesis. High values of $\alpha /$ Fe result from star formation that occurs before type 1a supernovae significantly increase the abundance of $\mathrm{Fe}$ in the interstellar medium, and are expected mainly in metal-poor environments. In M87, we find that the opposite is true. We use $\mathrm{Mgb} / \mathrm{Fe}=2 \mathrm{Mgb} /(\mathrm{Fe} 5270+\mathrm{Fe} 5335)$ as an indicator of $\alpha / \mathrm{Fe}$ and calibrate this indicator with the stellar population models of Thomas et al. (2011). As shown on Figure 9, the light-element abundance increases with metallicity. For G1 

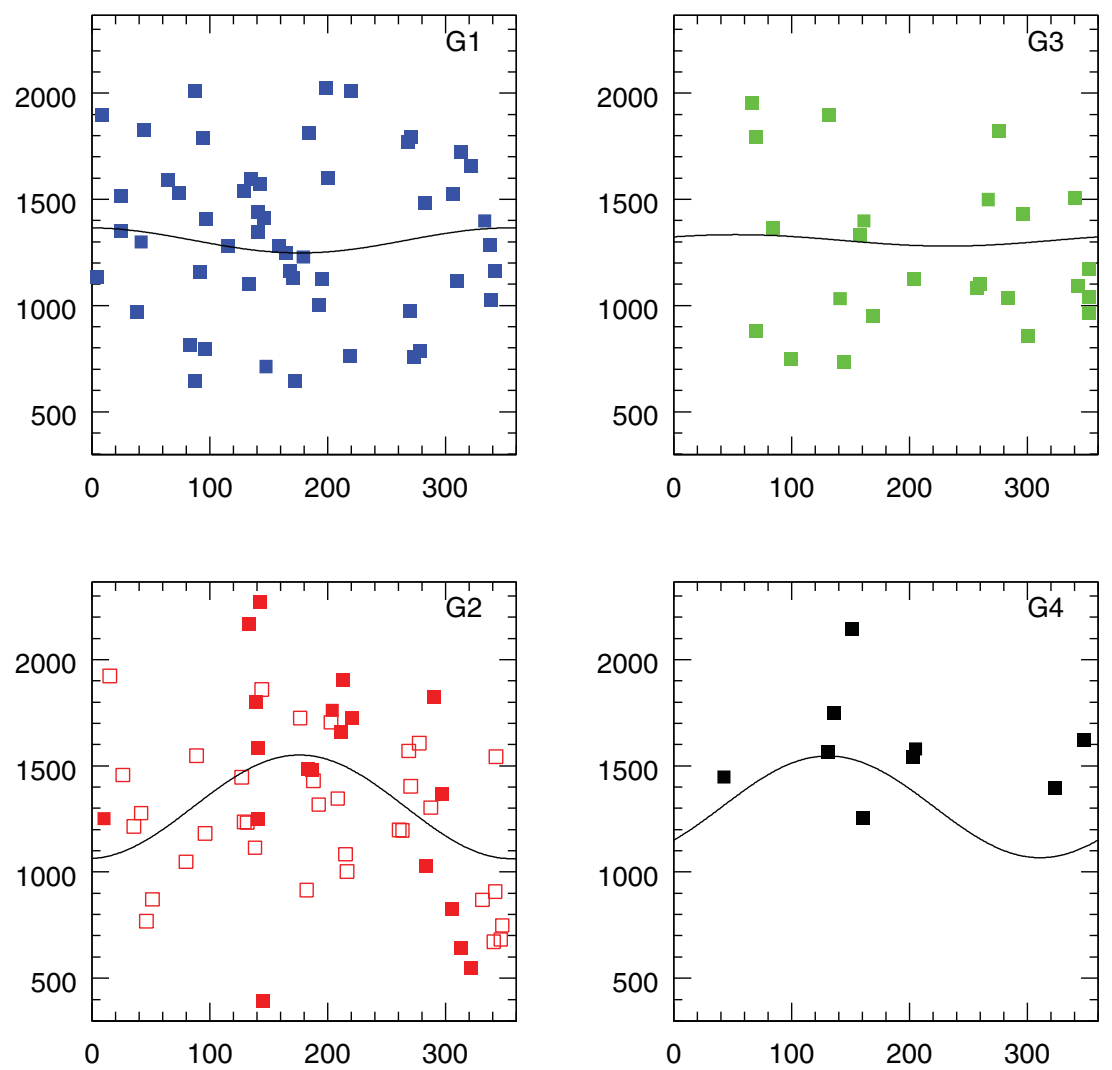

Figure 10. $v_{\text {rad }}$ versus PA with fitted mean rotation curves for G1-G4. In the box for G2, the red open squares are for $R \leq 300 \operatorname{arcsec}$ and the red full squares are for $R>300 \operatorname{arcsec}$. The outer GCs of that group rotate faster than the inner GCs

and $\mathrm{G} 2$ it increases from 0 to 0.3 , and for G4 it is about 0.4 . For G3, which is very metal-poor, the disagreement between $\alpha / \mathrm{Fe}$ and its indicator $\mathrm{Mgb} / \mathrm{Fe}$ suggests that the light-element ratio estimates, which are more uncertain at low metallicity, are just not reliable for this group, which is ignored. The contrast between the GCs of M87 and those of our Galaxy and M31 in this respect are further evidence that GCs of elliptical and spiral galaxies have different chemical histories.

We have adjusted a mean rotation curve to each of the four groups (see Section 3.4). The resulting parameters are listed in Table 2, and the mean rotation curves of the four groups along with radial velocities of the GCs are shown in Figure 10. The only groups with clear evidence of rotation are G2 and G4. The outer (radius > 300 arcsec) clusters of the group $\mathrm{G} 2$ rotate faster than the inner ones. Schuberth et al. (2010) have found that the metal poor GCs in the outer region of NGC 1399 (the non-rotating giant elliptical galaxy in the centre of the Fornax cluster) is the only GC population that shows significant rotation. The difference with M87 is that $\mathrm{G} 2$ is not the metal poorest group. There is marginal evidence for rotation in G1, about an axis which is orthogonal to that of G2. There is also evidence for rotation in G4, whose spatial distribution is flattened in the plane of rotation (along position angles of maximum or minimum radial velocity; see Figure 10). As G4 is centrally concentrated, it is worth mentioning the recent study of Emsellem et al. (2007), who find a counter-rotating core in the central 2 arcsec of M87, in position angle 20 degrees. In a detailed kinematic study of the GCs of M87, Strader et al. (2011) have found subpopulations with distinct kinematical behaviours, and concluded that M87 is still in active assembly. They also warned that the old kinematical data (which include the present ones) predict a higher rotation than their new data. Our own kinematical results should thus be viewed with caution.

\subsection{Origin of the four groups}

The first stage of formation of a galaxy is a dissipative collapse. During that phase, chemical enrichment is expected to be accompanied by spin up of the metal-richer material which ends up in circular orbits, whereas the metal-poor material is in eccentric orbits (Eggen, Lynden-Bell, \& Sandage 1962). The formation of G3 and G4 can be associated to that phase : G3 in the outer parts from metal-poor material, G4 in the inner part from metal-rich material. The lack of rotation in G3, the flattened spatial distribution, strong rotation and high average $\alpha / \mathrm{Fe}$ in $\mathrm{G} 4$, are properties consistent with this assumption. Montes et al. (2014a) have shown that the central part of M87 is super solar and presumably formed first during the monolithic collapse of the galaxy.

Montes et al. (2014b) have found that the innermost GCs of M87 (within $30 \mathrm{arcsec}$ ) are metal poorer than the stars in 
the region. We show that there are actually two populations of GCs (G3 and G4) near the centre, one of solar metallicity like the stars of the central region (Montes et al. 2014a).

In the second stage of formation, the galaxy accretes lowmass satellites in a dissipationless fashion (Bekki \& Chiba 2005). The GCs of G1 and G2 may have formed in this phase. The difference between G1 and G2 could be one of time, G2 forming first and retaining the angular momentum of the gas from which it formed and G1 forming later from cooling flows close to the centre, the gas having lost its angular momentum to collision with gas clouds (viz. Table 2, $v_{\text {rot }}$ ). This would explain why the GCs of G1, near the centre, have little rotation, while those of $\mathrm{G} 2$, in the outer regions, rotate significantly. In this scenario, one thus expects the velocity of rotation to increase outward, which seems to be the case for the GCs of G2: $v_{\text {rot }} \sim 374.93 \mathrm{~km} \mathrm{~s}^{-1}$ for $R \geq 300$, and $v_{\text {rot }} \sim 179.92 \mathrm{~km} \mathrm{~s}^{-1}$ ) for $R<300$.

\section{CONCLUSION}

We have performed a multivariate analysis of the chemical and photometric properties of the GCs of M87, and found four groups of clusters with distinct properties. The latter are used to propose a formation scenario for the different groups, in terms of dissipational collapse of the galaxy, followed by dissipationless accretion of matter.

Regardless of the clustering results, the reanalysis of data from the literature with recent stellar population models, those of Thomas et al. (2011) and the Yonsei models (Chung et al. 2013), provides new insights into the chemical properties of the GCs of M87, compared to those of other galaxies. We have found that these properties are different from those of GCs in M31, in that the Lick indices $\mathrm{NaD}$ and TiO1 are higher at a given $\mathrm{Mgb}$. We have also found a progressive excess blueing of the GCs with metallicity, which we interpret by the presence of an extreme blue HB, possibly due to He enriched secondary stellar populations. Finally, $\alpha / \mathrm{Fe}$ increases with metallicity, at variance with what occurs in GCs of spiral galaxies (our own and M31). Our interpretation is one among many others and remains speculative in view of the smallness of the sample and is not an ambitious as is needed. Our interpretation is only one possible way of understanding the results.

\section{ACKNOWLEDGEMENTS}

We thank C. Chung for sending us tables for the Yonsei Evolutionary Population Synthesis models. We are also thankful to A.K.Chattopadhyay for useful discussions, and to Saptarshi Mondal for helping in some calculations. One of the authors (Tanuka Chattopadhyay) thanks DST, India for providing her a major research project for the work.

\section{REFERENCES}

Albazzaz, H., \& Wang, X. Z. 2004, Industrial \& Engineering Chemistry Research, $43(21),{ }^{6731}$
Babu, G. J., Chattopadhyay, T., Chattopadhyay, A. K., \& Mondal, S. 2009, ApJ, 700, 1768

Bekki, K., \& Chiba, M. 2005, ApJ, 625, L107

Betoule, M., et al. 2013, A\&A, 552, A124

Brodie, J. P., \& Strader, J. 2006, ARA\&A, 44, 193

Chattopadhyay, A. K., Chattopadhyay, T., Davoust, E., \& Mondal, S. 2009, ApJ, 705, 1533

Chattopadhyay, A. K., Mondal, S., \& Chattopadhyay, T. 2013, CSDA, 57, 17

Chattopadhyay, T., \& Chattopadhyay, A. 2007, A\&A, 472, 131

Chattopadhyay, T., \& Karmakar, P. 2013, NewA, 22, 22

Chattopadhyay, T., Sharina, M., Davoust, E., De, T., \& Chattopadhyay, A. K. 2012, ApJ, 750, 91

Chattopadhyay, T., Sharina, M., \& Karmakar, P. 2010, ApJ, 724, 678

Chung, C., Lee, S. Y., Yoon, S. K., \& Lee, Y. W. 2013, ApJ, 769, L3

Cohen, J. G., Blakeslee, J. P., \& Côté, P. 2003, ApJ 592, 866

Cohen, J. G., Blakeslee, J. P., \& Ryzhov, A. 1998, ApJ, 808, 539

Cohen, J. G., \& Ryzhov, A. 1997, ApJ, 486, 230

Colucci, J. E., Bernstein, R. A., Cameron, S. A., \& McWilliam, A. 2012a, ApJ, 746, 29

Colucci, J. E., Bernstein, R. A., \& Cohen, J. 2012b, Proc. XII International Symp. on Nuclei in the Cosmos, 99

Comon, P. 1994, SigPr, 36, 287

Côté, P., et al. 2001, ApJ, 559, 828

Eggen, O. J., Lynden-Bell, D., \& Sandage, A. R. 1962, ApJ, 136, 748

Emsellem, E., et al. 2007, MNRAS, 379, 401

Fraix-Burnet, D., Chattopadhyay, T., Chattopadhyay, A. K., \& Davoust, E. 2010, MNRAS, 407, 2207

Fraix-Burnet, D., Davoust, E., \& Charbonnel, C. 2009, MNRAS, 398, 1706

Hanes, D. A., Cöté, P., Bridges, T. J., McLaughlin, D. E., Geisler, D., Harris, G. L. H., Hesser, J. E., \& Lee, M. G. 2001, ApJ, 559, 812

Huchra, J. P., \& Brodie, J. P. 1987, AJ, 93, 779

Jesseit, R., Cappellari, M., Naab, T., Emsellem, E., \& Burkert, A. 2009, MNRAS, 397, 1202

Johnson, R. A., \& Wichern, D. W. 1998, Applied Multivariate Statistical Analysis (4th edn.; Upper Saddle River: Prentice Hall)

Kaviraj, S., Sohn, S. T., O'Connell, R. W., Yoon, S.-J., Lee, Y. W., \& Yi, S. K. 2007, MNRAS, 377, 987

Kotulla, R., Fritze, U., Weilbacher, P., \& Anders, P. 2009, MNRAS, 396, 462

Krajnovic, D., et al. 2008, MNRAS, 390.93

Kissler-Patig, M., \& Gebhardt, K. 1998, AJ, 116, 2237

Lada, C. J., \& Lada, E. A. 2003, ARA\&A, 41, 57

Lambert, S. B., \& Gontier, A.-M. 2009, A\&A, 493, 317

Levenberg, K. 1944, QApMa, 2, 164

MacQueen, J. 1967, in Proc 5th Berkeley Symp. Math Prob. Vol.1, ed. L. M. Lecom \& J. Neyman (Los Angeles: University of California Press), 281

Maratson, C., Greggiol, L., Renzini, A., Ortolani, S., Saglia, R. P., Puzia, T. H., \& Kissler-Patig, M. 2003, A\&A, 400, 823

Marquardt, D. W. 1963, SIAMJ, 11, 431

Martell, S. L., \& Grebel, E. K. 2010, A\&A, 519, 14

Montes, M., Trujillo, I., Prieto, M. A., \& Acosta-Pulido, J. A. 2014a, MNRAS, 439, 990 
Montes, M., Trujillo, I., Prieto, M. A., \& Acosta-Pulido, J. A. 2014b, MNRAS, 439, 990

Mould, J. R., Oke, J. B., De Zeeuw, P. T., \& Nemec, J. M. 1990, AJ, 99, 1823

Mould, J. R., Oke, J. B., \& Nemec, J. M. 1987, AJ, 93, 53

Muratov, A. L., \& Gnedin, O. Y. 2010, ApJ, 718, 1266

Park, H. S., Lee, M. G., Hwang, H. S., Kim, S. C., Arimoto, N., Yamada, Y., Tamura, N., \& Onodera, M. 2012, ApJ, 759, 116

Proctor, R. N., Forbes, D. A., Romanwosky, A. J., Brodie, J. P., Strader, J., Spolaor, M., Mendel, J. T., \& Spitler, L. 2009, MNRAS, 398, 91

Richtler, T., et al. 2004, AJ, 127, 2094

Schiavon, R. P., Caldwell, N., Morrison, H., Harding, P., Courteau, S., MacArthur, L. A., \& Graves, G. J. 2012, AJ, 143, 14
Schuberth, Y., Richtler, T., Hilker, M., Dirsch, B., Bassino, L. P., Romanowsky, A. J., \& Infante, L. 2010, A\&A, 513, A52

Shapiro, S. S., \& Wilk, M. B. 1965, Biometrika, 52, 591

Sohn, S. T., O'Connell, R. W., Kundu, A., Landsman, W. B., Burstein, D., Bohlin, R. C., Frogel, J. A., \& Rose, J. A. 2006, AJ, 131, 866

Strom, S. E., Strom, K. M., Wells, D. C., Forte, J. C., Smith, M. G., \& Harris, W. E. 1981, ApJ, 245, 416

Strader, J., et al. 2011, ApJS, 197, 33

Sugar, A. S., \& James, G. M. 2003, J. Am. Stat. Assoc., 98, 750

Thomas, D., Maraston, C., \& Johansson, J. 2011, MNRAS, 412, 2183

Woodley, K. A., Harris, W. E., Beasley, M. A., Peng, E. W., Bridges, T. J., Forbes, D. A., \& Harris, G. L. H. 2007, AJ, 134, 494 